\section{Characteristics and outcomes of paediatric rhegmatogenous retinal detachment treated by segmental scleral buckling plus an encircling element}

\begin{abstract}
Purpose To evaluate the outcome of paediatric rhegmatogenous retinal detachment treated by segmental scleral buckling plus an encircling element.

Methods A retrospective review was carried out of 15 consecutive paediatric patients (16 eyes).

Results Thirteen of the 15 patients were male $(87 \%), 2(13 \%)$ were female. Their average age was 10.9 years (range $6-18$ years; median 10 years). Retinal detachment was caused by trauma in 9 of 16 eyes (56\%), high myopia in 5 of 16 eyes $(31 \%)$ and had an unknown cause in 2 of 16 eyes (13\%). Diagnosis was delayed by more than 1 month in 10 of 16 eyes $(63 \%)$. The visual acuity was $6 / 60$ or worse and the macula was detached on presentation in 13 of 16 eyes (81\%). Two of 16 eyes (13\%) had mild proliferative vitreoretinopathy. Final reattachment was achieved in all cases. Of 11 eyes with a follow-up of 6 months or more, there was improvement in visual acuity in 7 $(63 \%)$, and a best-corrected visual acuity of $6 / 20$ or better in $5(46 \%)$.

Conclusion Paediatric rhegmatogenous retinal detachment is characterised by a delay in diagnosis and a high degree of macular involvement on presentation. Anatomical reattachment with segmental scleral buckling plus an encircling element was successful in all eyes, and improvement of visual acuity was achieved in one-half of the eyes which had a follow-up of 6 months or more.
\end{abstract}

Key words Detachment, Paediatric, Retina, Scleral buckling

Paediatric rhegmatogenous retinal detachment is a rare condition, estimated to occur in 2.9/ 100000 children per year. ${ }^{1}$ However, by bearing such a grave prognosis for vision in young patients, it is a serious long-term problem. The
ALON D. SADEH, GAD DOTAN, RIVKA BRACHA $\dagger$, MOSHE LAZAR, ANAT LOEWENSTEIN aetiology, natural history and treatment of rhegmatogenous retinal detachment in adults are well known. However, the features of this condition in children have not been clearly determined, with the age limit of published reports varying from 14 to 21 years of age and the number of reported paediatric cases being relatively small. ${ }^{2-5}$ Surgical methods used in the treatment of retinal detachment in children include scleral buckling procedures (partial and encircling $)^{2,4,5}$ with or without vitrectomy. ${ }^{6,7}$ Okinami et al. ${ }^{8}$ used the encircling procedure for the primary operation in $26 \%$ of their 860 juvenile operated cases, and concluded that it is unavoidable for securely reattaching the retina.

We are aware of no study that has focused specifically on surgical and visual function in paediatric cases that required an encircling scleral buckling. The aim of this study was to evaluate the anatomical and functional outcomes of retinal detachment treated by segmental scleral buckling plus an encircling element in children.

\section{Methods}

We reviewed the files of all cases of rhegmatogenous retinal detachment (RRD) in children (up to 18 years old) who were treated by segmental scleral buckling plus an encircling element by a single surgeon (R.B.) in the Tel-Aviv Sourasky Medical Center between 1990 and 1998. Sixteen eyes of 15 children were identified and included in the study. Four cases for which sufficient follow-up was not available (at least 3 months follow-up) were not included in this study. The following data were retrieved from the medical files: sex, age at time of diagnosis, the aetiology of retinal detachment, visual acuity and macular involvement on presentation, and the elapsed time since the onset of the symptoms until the diagnosis was made. The existence and nature of proliferative vitreoretinopathy (PVR) were retrieved from

\author{
A.D. Sadeh \\ G. Dotan \\ M. Lazar \\ A. Loewenstein \\ Department of \\ Ophthalmology \\ Tel-Aviv Sourasky Medical \\ Center \\ and \\ Sackler Faculty of Medicine \\ Tel-Aviv, Israel
}

Anat Loewenstein, MD Department of

Ophthalmology

Tel-Aviv Medical Center

6 Weitzman Street

Tel-Aviv 64239, Israel

Tel: +97236973408

Fax: +97236917427

e-mail: stevelow@actcom.co.il

†Deceased

Received: 17 January 2000 Accepted in revised form: 15 August 2000 


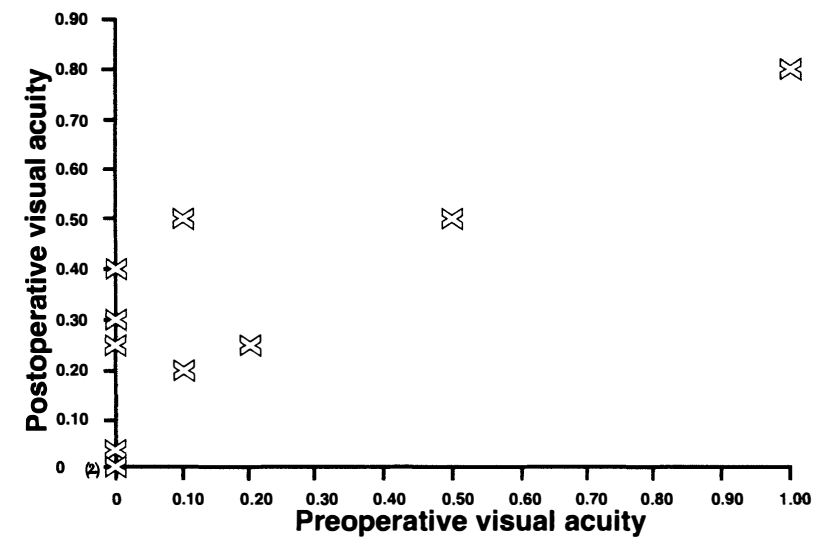

Fig. 1. Scatter graph comparing pre-operative and post-operative visual acuity in 11 eyes with at least 6 months of follow-up. (Visual acuity of light perception was arbitrarily defined as 1/1000, that of hand movement as 1/500 and that of counting fingers as 1/250.)

the chart and reclassified according to the Retina Society classification. ${ }^{9}$ As a rule, all our paediatric RRDs were encircled. We reviewed the outpatient clinic files of these patients to retrieve data on the results of the operation, recurrence of retinal detachment and final visual acuity.

\section{Results}

Sixteen eyes of 15 children were included in the study. Thirteen of the 15 patients were male $(87 \%)$. The average age of the study group was 10.9 years (range 6-18 years, median 10 years). The most common cause of retinal detachment was blunt trauma, in 9 of 16 eyes (56\%); 5 of these cases $(56 \%)$ were dialysis detachments. High myopia (greater than -6.00 dioptres) was the cause in 5 of 16 cases $(31 \%)$; none of these patients had any systemic disorder. The aetiology of the retinal detachment was unknown in 2 of 16 patients (13\%).

Visual acuity on presentation was $6 / 30$ or better in 3 of 16 eyes (19\%) and 6/60 or worse in the other 13 of 16 $(81 \%)$. The macula of these 13 eyes (with visual acuity of $\leqslant 6 / 60$ ) was detached on presentation. In 10 of 16 eyes $(63 \%)$, the duration from onset of symptoms until diagnosis of retinal detachment was more than a month (average 65 days, range 3 days to 4 years, median 62 days for the whole study group). There was mild PVR (grade A or B) on presentation in 2 of $10(20 \%)$ eyes.

The surgical procedure in all patients consisted of cryopexy of the retinal tear, and suturing a segmental 277 explant (7 mm width) and a 240 ( $2.5 \mathrm{~mm}$ width) band. Fourteen of 16 eyes $(88 \%)$ underwent one operation and the other $2(13 \%)$ were subsequently re-operated on due to recurrent retinal detachment. Final reattachment was achieved in all operated eyes. The average follow-up was 50 months (range 1 month to 10 years, median 56 months).

Final visual acuity at 6 months or more after surgery was available for 11 of 16 eyes (69\%) (5 other cases were lost to follow-up for various reasons). The post-operative improvement in visual acuity in these patients is demonstrated in Fig. 1: it was achieved in 7 of 11 eyes $(67 \%)$, and was recorded as being $6 / 20$ or better in 5 eyes $(46 \%)$. Of the 3 eyes with attached macula on presentation, $2(67 \%)$ achieved a visual acuity of $6 / 20$ or better. However, of the 8 eyes with detached macula on presentation, only $3(38 \%)$ achieved a visual acuity of $6 / 20$ or better, and $3(38 \%)$ achieved a visual acuity of $6 / 60$ or worse.

\section{Discussion}

The aim of this study was to evaluate the anatomical and functional outcomes of paediatric rhegmatogenous retinal detachment treated by segmental scleral buckling plus an encircling element. Final anatomical reattachment was achieved in all cases. There was improvement in visual acuity in $64 \%$ of the patients for whom follow-up of 6 months or more was available, and visual acuity of $6 / 20$ or better was achieved in $46 \%$ of these patients. Five patients were lost from follow-up before 6 months from surgery had passed.

The features of paediatric rhegmatogenous retinal detachment have been only partially determined in previous studies and the long-term surgical and functional outcomes of segmental scleral buckling plus an encircling element, specific to the juvenile population, have not previously been reported. Although encircling scleral buckling was described as being unavoidable in paediatric cases, ${ }^{8}$ one cannot ignore the potential disadvantages of an encircling element (including the use of more scleral sutures, increased intraocular pressure and optical refraction changes). Kreissig et al. ${ }^{10}$ challenged those surgeons who use techniques that include encircling the eye; however, their study did not focus on paediatric cases.

Although it is a rare condition, retinal detachment in children may have grave long-term implications. Our results indicate, however, that the long-term prognosis of paediatric retinal detachment treated by circling scleral buckling is fair when there is no or mild PVR.

In our study, 13 of the 15 patients were male (87\%). This proportion is in accordance with other studies. ${ }^{2,8}$ The aetiology of paediatric rhegmatogenous retinal detachment is different from the aetiology of adult cases.

Table 1. Pre-operative characteristics of paediatric rhegmatogenous retinal detachments in the literature and the current series

\begin{tabular}{|c|c|c|c|c|}
\hline & Bodard et al. ${ }^{2}$ & Lemrini et al. ${ }^{4}$ & Ouertani et al. ${ }^{3}$ & Current series \\
\hline Year & 1978 & 1993 & 1994 & \\
\hline No. of eyes & 37 & 30 & 24 & 16 \\
\hline Traumatic aetiology & $44 \%$ & $28 \%$ & $36 \%$ & $56 \%$ \\
\hline Detached macula & $77 \%$ & NA & $92 \%$ & $81 \%$ \\
\hline
\end{tabular}

NA, not available. 
Table 2. Post-operative characteristics of paediatric rhegmatogenous retinal detachments in the literature and the current series

\begin{tabular}{|c|c|c|c|c|}
\hline & Bodard et al. ${ }^{2}$ & Lemrini et al. ${ }^{4}$ & Ouertani et al. ${ }^{3}$ & Current series \\
\hline Year & 1978 & 1993 & 1994 & \\
\hline No. of eyes & 37 & 30 & 24 & 16 \\
\hline Anatomical success & $75 \%$ & $77 \%$ & $78 \%$ & $100 \%$ \\
\hline Visual acuity & $\geqslant 6 / 30$ in $40 \%$ & $\geqslant 6 / 10$ in $41 \%$ & $\geqslant 6 / 30$ in $24 \%$ & $\geqslant 6 / 20$ in $45 \%$ \\
\hline
\end{tabular}

${ }^{a}$ Various buckling techniques were used. No association between specific technique and results is given.

In our study, the cause of retinal detachment was blunt trauma in 9 of 16 eyes (56\%) and high myopia in 5 of 16 (31\%). In a study that included 908 eyes, Okinami et al. ${ }^{8}$ found that retinal detachments in patients of 0-9 years of age were different from those of patients of 10-19 years in many respects, including an abundance of traumatic detachments in the younger group ( $42 \%$ vs $27 \%$ ). Rosner et al. ${ }^{1}$ reported that in as many as $62 \%$ of their study group of 13 patients, retinal detachment was related to ocular trauma. Lower rates of cases related to trauma were reported in other studies (Table 1). ${ }^{2-5}$ It is noteworthy that there was no statistically significant association between traumatic aetiology and postoperative reattachment rates in the large series by Okinami et al. ${ }^{8}$

There are a number of features of paediatric retinal detachment that might result in poor prognosis: high rate of macular involvement, late diagnosis and a high rate of PVR. In our study, the macula was detached on presentation in 13 eyes $(81 \%)$. This finding is in accordance with previous reports (Table 1). ${ }^{2-5}$ In 10 eyes $(63 \%)$, the time interval between the onset of symptoms until diagnosis of retinal detachment was more than 1 month. Bodard et al. ${ }^{2}$ reported a delay of 3 months in $48 \%$ of their cases, and suggested that this may be the result of several factors, including the child being afraid to report his or her visual impairment.

The anatomical operative results of our patients were excellent: 14 of 16 eyes underwent one operation $(88 \%)$ and 2 of $16(13 \%)$ were later re-operated on due to recurrent retinal detachment. Final re-attachment was achieved in all operated eyes. These results are better than the previously reported data for scleral buckling procedures (Table 2 ).$^{3-5}$ It is noteworthy, however, that previous studies included various buckling techniques.

In our study, the improvement in visual acuity following surgery was found in $64 \%$ of patients for whom follow-up of 6 months and more was available. Visual acuity of $6 / 20$ or better was achieved in $46 \%$ of cases. Although it is hard to compare functional results between various reports due to the use of different scales, it seems that previously reported results were somewhat less successful than ours (Table 2). ${ }^{3-5}$ A possible explanation of this finding is that the use of an encircling buckle lowers the traction on the vitreous base, thus lowering the possibility of subsequent PVR development. Limitations that should be mentioned about our study was the small number of patients, the limited follow-up period of some of the patients, and the loss of 5 cases from long-term follow-up.

Although numerous negative factors are associated with paediatric rhegmatogenous retinal detachment (traumatic aetiology, delayed diagnosis and detached macula on presentation), good recuperation can be expected when no significant PVR has developed.

\section{References}

1. Rosner M, Treister G, Belkin M. Epidemiology of retinal detachment in childhood and adolescence. J Pediatr Ophthalmol Strabismus 1987;24:42-4.

2. Bodard GE, Pasticier A, Biojout G, Forest A. Prognosis of retinal detachment in children [in French]. J Fr Ophtalmol 1978;1:589-95.

3. Ouertani A, Zhioua R, el Euch M, Mili I. Retinal detachment in young patients [in French]. J Fr Ophtalmol 1994;17:388-93.

4. Lemrini F, Dafrallah L, Kabbaj A. Retinal detachment in children [in French]. J Fr Ophtalmol 1993;16:159-64.

5. Winslow RL, Tasman W. Juvenile rhegmatogenous retinal detachment. Ophthalmology 1978;85:607-18.

6. Ferrone PJ, McCuen BW, de Juan E, Machemer R. The efficacy of silicone oil for complicated retinal detachments in the pediatric population. Arch Ophthalmol 1994;112:773-7.

7. Moisseiev J, Vidne O, Treister G. Vitrectomy and silicone oil injection in pediatric patients. Retina 1998;18:221-7.

8. Okinami S, Ogino N, Nishimura T, Tano Y. Juvenile retinal detachment. Ophthalmologica 1987;194:95-102.

9. Machemer R, Aaberg TM, Freeman HM, Irvine AR, Lean LS, Michels RM. An updated classification of retinal detachment with proliferative vitreoretinopathy. Am J Ophthalmol 1991;112:159-65.

10. Kreissig I, Simader E, Fahle M, Lincoff H. Visual acuity after segmental buckling and non-drainage: a 15-year follow-up. Eur J Ophthalmol 1995;5:240-6. 